\title{
PHILIPPA LEWIS, Intimacy and Distance. Conflicting \\ Cultures in Nineteenth-Century France
}

\section{Maria Emanuela Raffi}

\section{(2) OpenEdition}

\section{Journals}

\section{Edizione digitale}

URL: http://journals.openedition.org/studifrancesi/15658

DOI: ERREUR PDO dans /localdata/www-bin/Core/Core/Db/Db.class.php L.34 : SQLSTATE[HYO0O]

[2006] MySQL server has gone away

ISSN: 2421-5856

\section{Editore}

Rosenberg \& Sellier

\section{Edizione cartacea}

Data di pubblicazione: 1 décembre 2018

Paginazione: 516-517

ISSN: 0039-2944

\section{Notizia bibliografica digitale}

Maria Emanuela Raffi, «PHILIPPA LEWIS, Intimacy and Distance. Conflicting Cultures in Nineteenth-Century France», Studi Francesi [Online], 186 (LXII | III) | 2018, online dal 01 janvier 2019, consultato il 08 janvier 2021. URL: http://journals.openedition.org/studifrancesi/15658; DOI: https://doi.org/10.4000/ studifrancesi. 15658

Questo documento è stato generato automaticamente il 8 janvier 2021.

\section{$\Theta \oplus \Theta \Theta$}

Studi Francesi è distribuita con Licenza Creative Commons Attribuzione - Non commerciale - Non opere derivate 4.0 Internazionale. 


\title{
PHILIPPA LEWIS, Intimacy and Distance. Conflicting Cultures in Nineteenth- Century France
}

\author{
Maria Emanuela Raffi
}

\section{NOTIZIA}

PHILIPPA LEWIS, Intimacy and Distance. Conflicting Cultures in Nineteenth-Century France, Oxford, Legenda, 2017, 187 pp.

1 Accompagnato da una bibliografia veramente ricca e da un dettagliato indice dei nomi, il saggio di Philippa LEWIS si occupa nella prima parte dell'Intimacy, prendendo come punto di partenza un saggio di Henry James su Sainte-Beuve, in cui l'autore mostra il carattere "intimo" della scrittura come una caratteristica di una importante zona della letteratura francese del XIX secolo: "poésie intime, the roman intime, and the journal intime». I punti di focalizzazione storica dello studio sono soprattutto due-la Monarchia di luglio e il Secondo Impero-e l'autore che si colloca più significativamente fra questi due periodi, mostrando il passaggio dalla letteratura dell'intimità a quella dell'«indifferenza distante» appare chiaramente Baudelaire. Certo, soprattutto per quanto riguarda la prima metà del secolo, altri nomi di autori ricorrono nel saggio della Lewis; Lamartine, Victor Hugo, Sainte-Beuve, Stendhal e molti altri illustrano l'intimité come una caratteristica tipicamente francese, eredità tuttavia della Sensibility inglese del secolo precedente, nelle diverse forme letterarie. Passando ad esaminare più nel dettaglio il modo in cui l'intimità viene trattata dai vari autori, l'A. rileva l'imbarazzo e le difficoltà che accompagnano l'espressione di tale condizione presso molti autori, dato che secondo il modello maschile della cultura ottocentesca post-rivoluzionaria, ogni manifestazione di sentimenti risulta ridicola e inappropriata. Tuttavia le poesie di Baudelaire («Confession» in particolare), la Double vie di Asselineau e Vie, poésies et pensées de Joseph Delorme di Sainte-Beuve propongono 
confidenze e confessioni, che costituiscono un nuovo genere di «intimisme» derivato certo dalle Confessions di Rousseau ma non senza difficoltà ed esitazioni, dato che la Lewis mostra gli interessanti cambiamenti intervenuti nella poesia «Confession» dalla versione 'privata' per Mme Sabatier all'edizione pubblicata nelle Fleurs, dove si fa più forte la condanna dell'intimità e comincia ad apparire la 'feroce ironia' baudelairiana. Con la stessa circospezione, Fromentin rappresenta nel suo romanzo Dominique le confidenze fra il narratore e il personaggio, espressioni di un'amicizia più suggerita che narrata.

2 Il caso del Journal come genere intimo per eccellenza viene esaminato dall'A. in particolare per il diario della sorella di Maurice de Guérin, Eugénie, autrice di Journal, lettres et poèmes (1862), poi per i Memoranda di Barbey d'Aurevilly, anch'essi concepiti come ipotetica conversazione con Guérin, nei quali appare, come per Baudelaire, un'evidente ironia, che Barbey stesso definisce «un génie qui dispense de tous les autres».

Uno spazio particolare è riservato al poème en prose di Baudelaire «À une heure du matin», pubblicato prima nella «Presse», poi nei Journaux intimes, che presenta alcuni evidenti tratti di scrittura diaristica ed è giudicato dai vari critici come un testo intimo ma ironico («an illusion of intimacy mischievously concocted by the poet», Murphy), terapeutico (Stephen, Desjardins, Rilke), costruito secondo la retorica della preghiera.

Il quarto capitolo pone il problema del duplice e contraddittorio orientamento espresso dalla letteratura del xIX secolo: «the desire for home, on the one hand, and for distance or dépaysement, on the other». Vengono quindi presi in esame i testi che cercano il confronto e criticano la facile intimità con altre realtà, attraverso viaggi e considerazioni su altri paesi, soprattutto l'esotico Voyage en Égypte di Flaubert, il feroce e «belgophobe» La Belgique déshabillée di Baudelaire e l'esempio di «colonial intimacies» fornito da Un été dans le Sahara di Fromentin.

5 Nella parte successiva («Experiencing Art: Baudelaire's Intimate Criticism»), Philippa Lewis si occupa delle arti visive e del genre intime espresso dalle opere di Vuillard o di Ingres, ma soprattutto della critica d'arte nei Salons di Baudelaire e nell'Exposition universelle. Il Salon de 1846, in particolare, unisce Decamps e Delacroix nel concetto di intimità, seppure con modalità differenti - «when equated with the detail and 'local colour'»-e introduce la metafora del critico come viaggiatore in una concezione dell'arte che si modifica e si amplia, metafora che prenderà corpo nell'Exposition Universelle del 1855. In essa Baudelaire tratta come «a genuine possibility» non solo la comprensione, ma addirittura l'adozione di sensibilità diverse e straniere, considerando le diverse estetiche "as fragments of the same "beauté universelle"»; l'appassionarsi a nuove estetiche appare a Baudelaire come un segno di vitalità che va ad aggiungersi alla vitalità già esistente.

6 «What is the role of friendship in the critical process?». In «Literary Criticism and the Rhetoric of Friendship», sesta parte del saggio, viene approfondita la concezione stessa della critica letteraria che si va definendo nel corso del xIx secolo e il tipo di rapporti che essa esprime. I 'portraits' di Sainte-Beuve, punto di riferimento per la prima metà del secolo, mostrano già «a combination of penchant and method», fra elementi personali e professionali, che l'A. analizza e approfondisce, mostrando come «the trope of friendship» continui a funzionare nella critica letteraria, con diverse modalità, per tutto il secolo. 
7 Il volume si chiude con alcune considerazioni su «Baudelaire intime»; l'autore delle Fleurs, pur mantenendo il modello di Sainte-Beuve, utilizza nella critica letteraria e d'arte un altro tipo di rapporto personale, meno immaginario e più concreto, che gli permette «to highlight meetings and conversations with the subject of his criticism» di cui esalta le qualità intellettuali e spirituali, escludendo gli aspetti troppo clamorosi. In definitiva l'attrazione per l'intime in ambito letterario pervade secondo Philippa Lewis anche la seconda metà del secolo, «countering narratives which present the cultivation of detachement as the exemplary impulse of the age». 